\title{
FAS-670 gene polymorphism and cervical carcinogenesis risk: A meta-analysis
}

\author{
QIAOYING HUANG ${ }^{1 *}, \mathrm{JIE} \mathrm{WANG}^{2 *}$ and YANLING HU ${ }^{2,3}$ \\ ${ }^{1}$ Graduate School, ${ }^{2}$ Center for Genomic and Personalized Medicine and ${ }^{3}$ Medical Research Center, \\ Guangxi Medical University, Nanning, Guangxi Zhuang Autonomous Region 530021, P.R. China
}

Received July 08, 2013; Accepted August 16, 2013

DOI: $10.3892 /$ br.2013.159

\begin{abstract}
FAS is a cell surface receptor that plays an important role in the etiology of cancer. Previous studies on the association between FAS-670 polymorphism and cervical carcinogenesis failed to reach a consensus; therefore, this meta-analysis was conducted to estimate the association of FAS-670 polymorphism and the risk of cervical cancer. This meta-analysis included 10 studies on FAS-670 genotyping, including a total of 2,901 cases and 2,831 controls. The complete overdominant model was applied in our meta-analysis $[\mathrm{AB}$ vs. AA: odds ratio $(\mathrm{OR})=0.879,95 \%$ confidence interval (CI): 0.775-0.998, $\mathrm{P}=0.046$; $\mathrm{BB}$ vs. $\mathrm{AA}: \mathrm{OR}=0.903$, 95\% CI: $0.775-1.052, \mathrm{P}=0.190]$. The random effects $\mathrm{OR}$ was 1.13 (95\% CI: $\left.0.95-1.34, \mathrm{I}^{2}=52.7 \%, \mathrm{P}_{\text {heterogeneity }}=0.03\right)$. An ethnic subgroup analysis was subsequently performed. The OR for Asians was 1.25 (6 comparisons, 95\% CI: $1.05-1.48, \mathrm{I}^{2}=23.5 \%$, $\left.\mathrm{P}_{\text {heterogeneity }}=0.03\right)$, whereas for Caucasians, no significant association was observed between FAS-670 polymorphism and cervical carcinogenesis (4 comparisons, $\mathrm{OR}=0.96$, 95\% CI: $\left.0.75-1.24, \mathrm{I}^{2}=45.9 \%, \mathrm{P}_{\text {heterogeneity }}=0.14\right)$.
\end{abstract}

\section{Introduction}

Apoptosis is a physiological process that regulates normal homeostasis and alterations of the apoptosis-related genes are likely to contribute to the pathogenesis of malignant tumors (1-3) and autoimmune diseases (4). FAS is a type of cell surface apoptotic signal transmission receptor. When combined with its natural ligand CD95L to initiate the death signal cascade, the complex leads to apoptosis $(5,6)$. The human FAS gene is one of members of the tumor necrosis factor receptor superfamily (7) and is located in chromo-

Correspondence to: Dr Yanling Hu, Medical Research Center, Guangxi Medical University, 22 Shuangyong Road, Nanning, Guangxi Zhuang Autonomous Region 530021, P.R. China E-mail: ylhupost@163.com

${ }^{*}$ Contributed equally

Key words: FAS-670, cervical carcinogenesis, meta-analysis some 10q24.1, involving 9 exons and 8 introns. Previous studies (8-10) reported that the downregulation of FAS may result in resistance to death signals in several types of cancer. Nunobiki et al (11) reported that the transcriptional expression of the FAS gene was regulated by a number of genetic elements located in the $5^{\prime}$ upstream promoter region of the gene. In the promoter region, Huang et al (12) reported that the polymorphism involved an A-to-G substitution at the -670 nucleotide position in the enhancer region (FAS-670 $\mathrm{A}>\mathrm{G}$, rs1800682) and the heterozygous A/G alleles were observed in $52 \%$ of the normal population, with a frequency of the $\mathrm{G}$ and A alleles of 0.49 and 0.51 , respectively.

The FAS-670 polymorphism consists of the variant genotypes FAS-670 G/G and FAS-670 A/G and the wild-type A/A. The frequency range of FAS-670 A/A among healthy controls was reported to be $25.5-43.6 \%$ and the frequency of the homozygous $\mathrm{G} / \mathrm{G}$ variant $\sim 12 \%$, whereas the frequency range of the heterozygous $\mathrm{A} / \mathrm{G}$ was reported to be $44.2-60.5 \%(13,14)$.

Cervical cancer is the second most common cancer among women worldwide $(11,15)$, with a high incidence $(>80 \%)$ in developing compared to developed countries $(15,16)$. Cervical cancer is on the increase in Asia (17) and exhibits relatively higher incidence and mortality rates in Hungary compared to those in other European Union countries (18). Moreover, cervical cancer was reported to constitute $23.3 \%$ of all cancers among African women (19).

Human papillomavirus (HPV) is widely considered as the key etiological agent in cervical carcinogenesis. A meta-analysis of cross-sectional high-risk HPV type distribution in 115,789 HPV-positive women was performed, with HPV16 positivity in particular increasing steeply from normal/atypical squamous cells of undetermined significance/low-grade squamous intraepithelial lesion (LSIL)/cervical intraepithelial neoplasia (CIN)1 (20-28\%), through CIN2/high-grade squamous intraepithelial lesion (HSIL) (40/47\%) to CIN3/invasive cervical cancer $(58 / 63 \%)$ in different regions (20). Furthermore, previous epidemiological studies investigated the etiology of cervical cancer in order to recommend preventive measures to reduce the incidence of cervical carcinogenesis and identified certain important environmental factors. Cervical cancer is considered to be a multifactorial disease, with smoking and age being important etiological factors contributing to increased risk $(21,22)$. Therefore, genetic as well as environmental factors may contribute to cervical carcinogenesis. 
Previous studies, including the 10 studies that we included in the present meta-analysis, were conducted to estimate the incidence of cervical carcinogenesis in association with the FAS-670 polymorphism; however, a consensus was not reached (13,23-31). Zhang et al (32) conducted a meta-analysis on FAS promoter polymorphisms and cancer risk, but failed to demonstrate a significant association with FAS-670 polymorphism. Since then, no confirmed outcomes based on small sample sizes or potential publication bias from the previous studies was obtained. Therefore, an updating meta-analysis was performed, using the accumulated data, to re-examine the association between the risk of cervical carcinogenesis and FAS-670 polymorphism.

\section{Materials and methods}

Search strategy. A search for eligible studies was conducted in PubMed, Embase and HuGNet electronic databases, using the following key words and word combinations: 'uterine cervical neoplasm', 'cervical cancer', 'cervical', 'cervix', 'FAS' and 'FAS-670'. The last update of retrieval was March 25, 2012. The search was limited to English language papers. Additional studies were identified through the reference lists of the original studies. We selected the articles with more information regarding the origin of cases and controls and the ones with the largest number of subjects among the overlapping reports.

Selection and exclusion criteria. The detailed selection criteria were as follows: i) case-control studies evaluating the association between FAS-670 polymorphism and the risk of cervical carcinogenesis; ii) case population including patients with precancerous lesions and cervical cancer patients; iii) control population comprising healthy individuals and not malignant tumor patients. The exclusion criteria were as follows: i) if similar studies included overlapping populations, only the most recent articles were included and the remaining were excluded; ii) insufficient data; iii) Hardy-Weinberg equilibrium (HWE) did not reach statistical significance $(\mathrm{P}<0.05)$.

Data extraction. The information was extracted from the eligible studies, including first author, year of publication, ethnicity, area, sample size of cases and controls, source of cases and controls, mean age of cases and controls and genotype frequency in cases and controls.

Statistical analysis. The ORs with their corresponding 95\% CIs were used as the metric of choice. Based on the individual ORs, the pooled OR was estimated. First, we investigated the distribution of genotypes in the control groups under HWE to obtain evidence of population stratification (HWE; $\mathrm{P}>0.05$ ) (33). We also estimated the association with cervical carcinogenesis risk with a complete overdominant genotypic model $\left(\mathrm{G} / \mathrm{G}+\mathrm{A} / \mathrm{A}\right.$ vs. A/G). Second, to assess the $\mathrm{P}_{\text {heterogeneity }}$ among different studies, a statistical test for heterogeneity was conducted using the $\mathrm{I}^{2}$ statistic, with values between 0 and $100 \%$, with higher values leading to greater heterogeneity (no heterogeneity, $\mathrm{I}^{2}$ : 0-25\%; moderate heterogeneity, $\mathrm{I}^{2}: 25-50 \%$; significant heterogeneity, $\mathrm{I}^{2}: 50-75 \%$; and extreme heterogeneity, $\mathrm{I}^{2}: 75-100 \%$ ) (34). If the effect sizes were homogeneous among the studies, the fixed effects model was used to estimate the overall effect size. Otherwise, a random effects model was used. Random effects may incorporate an estimate of between-study variance to a great extent and provide wider $95 \%$ CI.

To further investigate the source of heterogeneity, we performed a subgroup analysis by grouping studies with similar characteristics, such as ethnicity and sample size. The ethnic subgroups were categorized into Caucasian and Asian. In addition, a sensitivity analysis was employed. In the sensitivity analysis, studies was excluded one at a time to determine the magnitude of their effect on the overall summary estimate (35). Finally, publication bias was assessed using funnel plots and Begg's rank correlation test (36). All the P-values were two-sided. The statistical analysis was performed using Metagen and Stata software, version 11.0 (Stata Corp, College Station, TX, USA).

\section{Results}

Identification and characteristics. A total of 140 abstracts were retrieved through searching PubMed, Embase and HuGNet databases. We identified 16 relevant studies that described the association between the FAS-670 polymorphism and cervical carcinogenesis. However, after reading the full articles, one study was excluded as a letter (37), one as a review (11) and two due to the lack of raw data $(5,38)$. Two studies were overlapped $(13,39)$ and one was retained $(13)$ according to the criteria mentioned above. After calculating the HWE for each of the remaining studies, one more was excluded (14) and a total of 10 studies were finally included in this meta-analysis.

All the articles were case-control studies. Among the eligible studies, 6 were conducted on Asian $(13,24,25,27,28,30)$ and 4 on Caucasian populations $(23,26,29,31)$. Two studies were classified as LSIL and HSIL $(28,30)$ and one study included HSIL and cervical cancer (27), whereas others exclusively included cervical cancer patients $(13,23-26,29,31)$. Only one study reported the clinical stages (26). In all the studies, the majority of the patients were recruited from hospitals by blood samples or tissue specimens. Six studies mentioned the mean age of the patients $(13,23,24,27,29,30)$ and the remaining 4 studies did not $(25,26,28,31)$. All the studies used polymerase chain reaction. Other detailed information is presented in Table I.

Main results and subgroup analysis. In total, the eligible studies included 3,247 cases and 2,944 controls and a total of 2,901 cases and 2,831 controls were genotyped. The summary ORs and 95\% CIs for the FAS-670 polymorphism and the subgroup analysis are presented in Table II. The results indicated that FAS-670 was not associated with the risk of cervical carcinogenesis. The summary OR was 1.13 (95\% CI: 0.95-1.34), with between-study heterogeneity $\left(\mathrm{I}^{2}=52.7 \%, \mathrm{P}_{\text {heterogeneity }}=0.03\right)$. All the analyses were based on pooling of data from different populations. Therefore, a subgroup analysis according to different ethnicities was performed. The OR for Asians was 1.25 (6 comparisons, 95\% CI: $1.05-1.48, \mathrm{I}^{2}=23.5 \%, \mathrm{P}_{\text {heterogeneity }}=0.03$ ), whereas for Caucasians, no significant association was observed between FAS-670 polymorphism and the risk of cervical carcinogen- 


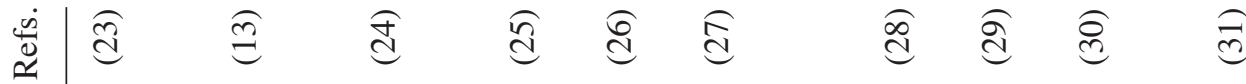

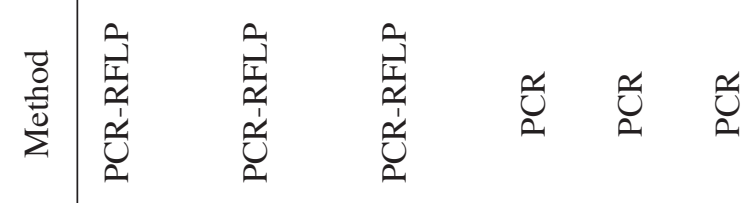

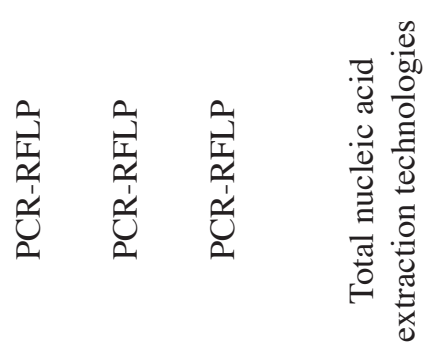

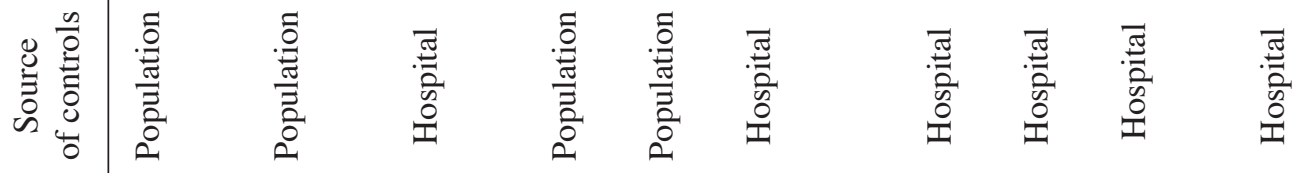

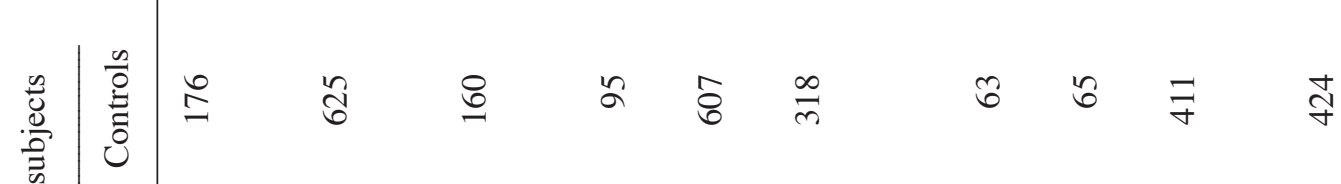

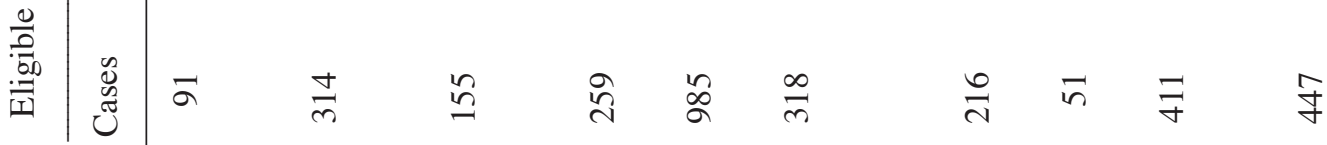

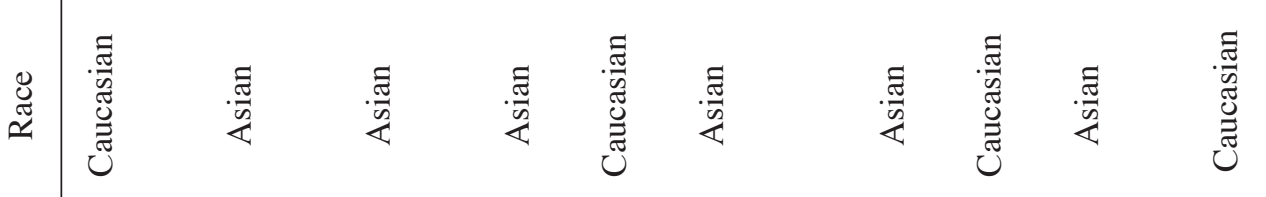


Table II. Summary ORs and 95\% CIs for FAS-670 polymorphism and subgroup analysis.

\begin{tabular}{lcccccr}
\hline $\begin{array}{l}\text { Subgroups } \\
\text { and FAS-670 } \\
\text { polymorphism }\end{array}$ & Comparisons (no.) & $\begin{array}{c}\text { Genotype } \\
\text { cases (no.) }\end{array}$ & $\begin{array}{c}\text { Genotype } \\
\text { controls (no.) }\end{array}$ & $\begin{array}{c}\text { Random effects } \\
\text { OR (95\% CI) }\end{array}$ & P $_{\text {heterogeneity }}$ & $\mathrm{I}^{2}(\%)$ \\
\hline $\begin{array}{l}\text { Population } \\
\text { Asians }\end{array}$ & 6 & 1,496 & 1,662 & $1.25(1.05,1.48)$ & 0.03 & 23.5 \\
$\quad \begin{array}{l}\text { Caucasians } \\
\text { Sample }\end{array}$ & 4 & 1,405 & 1,169 & $0.96(0.75,1.24)$ & 0.14 & 45.9 \\
$>200$ & 6 & 2,522 & 2,335 & $1.13(0.91,1.41)$ & 0.01 & 69 \\
$<200$ & 4 & 290 & 496 & $1.13(0.86,1.48)$ & 0.42 & 0.0 \\
Overall & 10 & 2,901 & 2,831 & $1.13(0.95,1.34)$ & 0.03 & 52.7 \\
\hline
\end{tabular}

OR, odds ratio; CI, confidence interval.

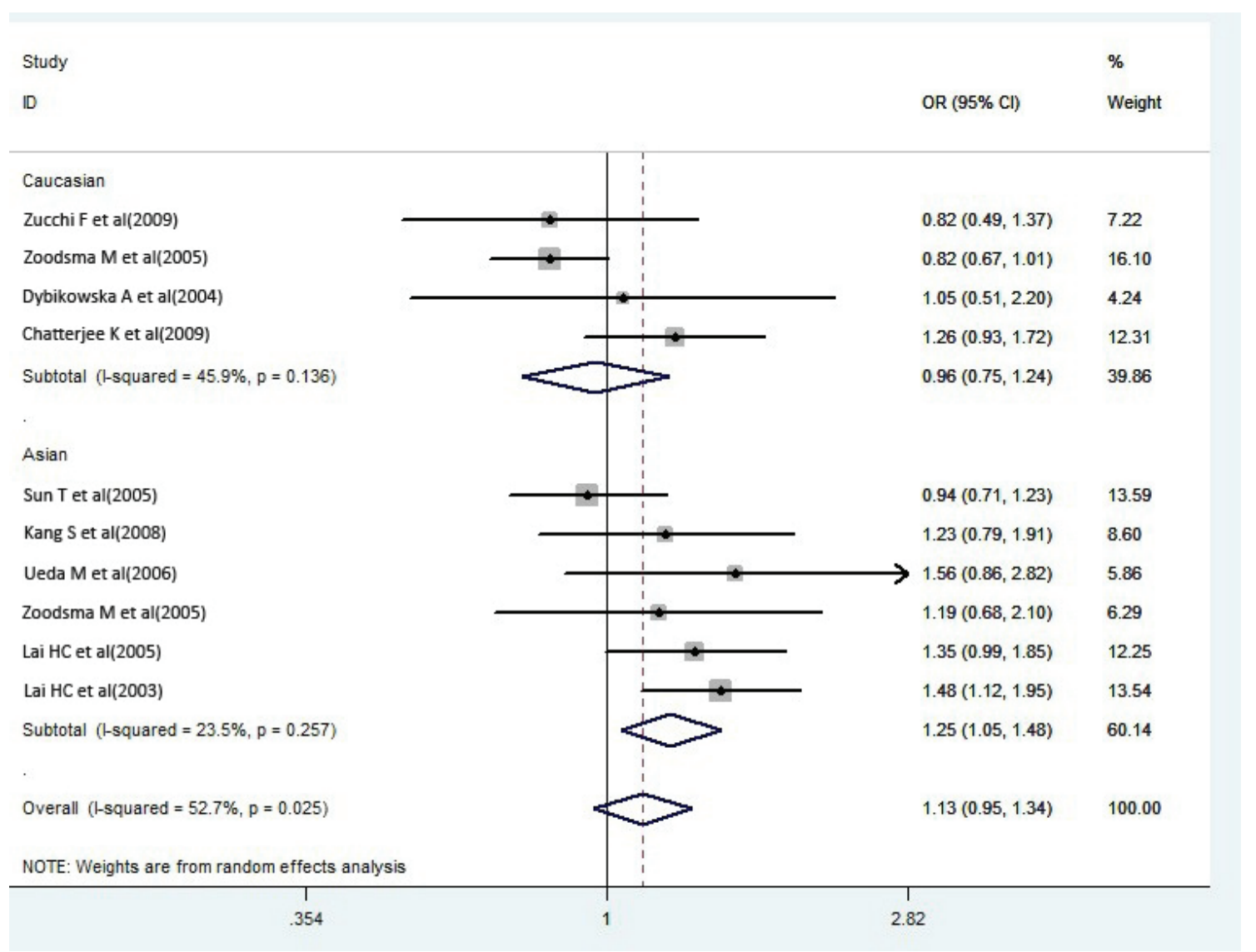

Figure 1. Effect of FAS-670 phenotype studies (first author, year of publication) on the risk of cervical cancer and combined estimate of odds ratio (OR) and $95 \%$ confidence interval (CI) by the complete overdominant effects model. Also shown is the summary random effects estimate for the comparison along with the respective $95 \%$ CI. Asian: Chi-squared test for heterogeneity $=6.54(\mathrm{df}=5), \mathrm{P}=0.257, \mathrm{I}^{2}=23.5 \%$; test of $\mathrm{OR}=1: \mathrm{z}=2.51$ ( $\left.\mathrm{P}=0.012\right)$. Caucasian: Chi-squared test for heterogeneity $=5.54(\mathrm{df}=3), \mathrm{P}=0.136, \mathrm{I}^{2}=45.9 \%$; test of $\mathrm{OR}=1: \mathrm{z}=0.3(\mathrm{P}=0.761)$. df, degree of freedom.

esis $\left(4\right.$ comparisons, $\mathrm{OR}=0.96,95 \% \mathrm{CI}: 0.75-1.24, \mathrm{I}^{2}=45.9 \%$, $\mathrm{P}_{\text {heterogeneity }}=0.14$ ) (Fig. 1).

In the sensitivity analysis, we applied the random effects model (Fig. 2) to estimate the risk of cervical cancer $(\mathrm{OR}=1.13$, 95\% CI: $0.95-1.34, \mathrm{I}^{2}=52.7 \%, \mathrm{P}_{\text {heterogeneity }}=0.03$ ). However, in the funnel plot analysis of publication bias, the funnel plot appeared to be symmetrical and the Egger's test $(\mathrm{P}=0.343)$ revealed no evidence of publication bias (Fig. 3).

\section{Discussion}

This meta-analysis, involving the comparison of a total of 3,247 cases and 2,944 controls, investigated 10 case-control studies on FAS-670 and assessed the association of FAS-670 polymorphism with the risk of cervical carcinogenesis. There was no significant evidence supporting an association between FAS-670 polymorphism and cervical cancer risk. 


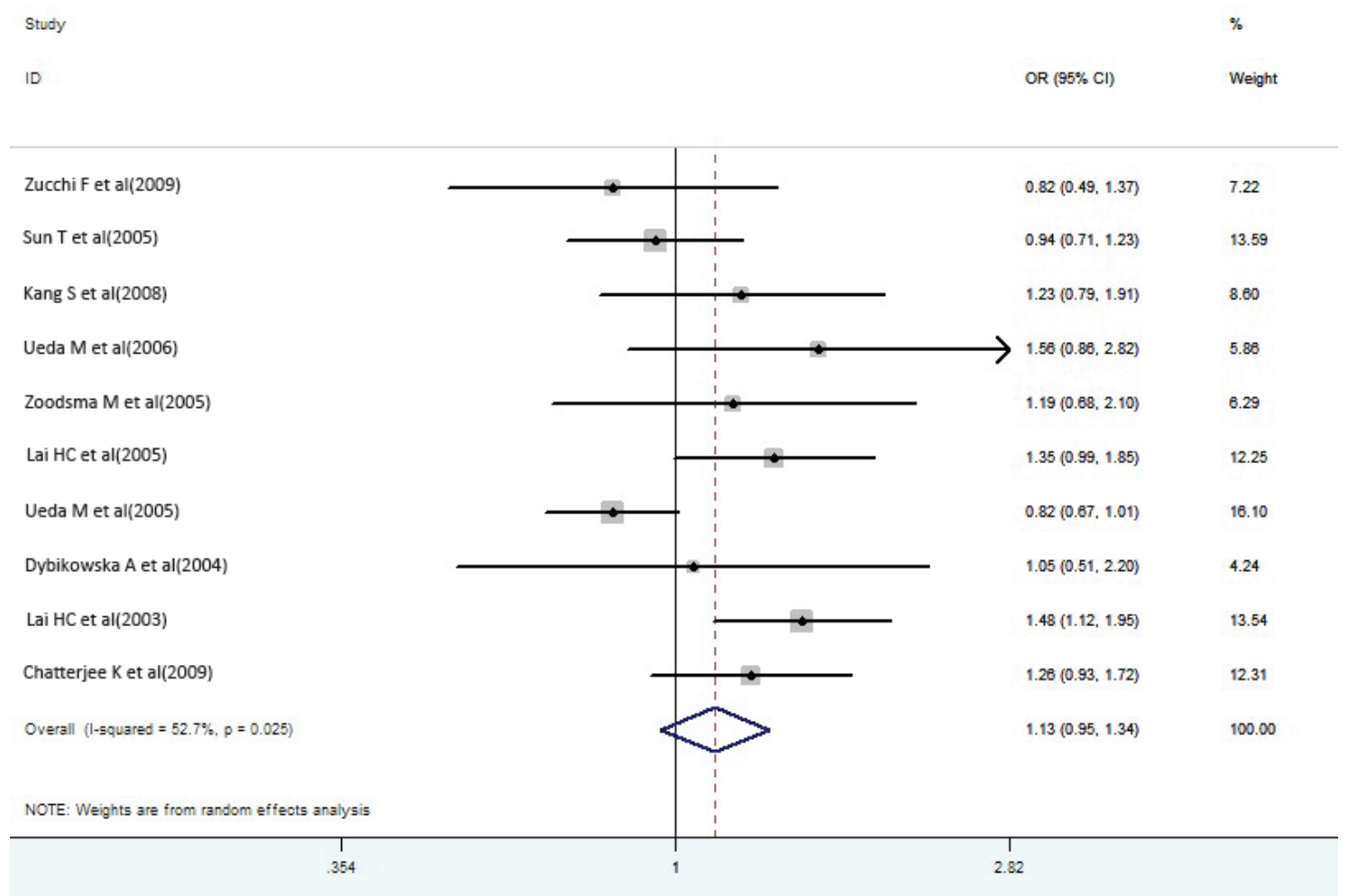

Figure 2. Summary plot for FAS-670 phenotype studies (first author, year of publication) and combined estimate of odds ratio (OR) and 95\% confidence interval $(\mathrm{CI})$ by the complete overdominant effects model. Chi-squared test for heterogeneity $=19.04(\mathrm{df}=9), \mathrm{P}=0.025, \mathrm{I}^{2}=52.7 \%$; test of $\mathrm{OR}=1: \mathrm{z}=1.40(\mathrm{P}=0.161)$.

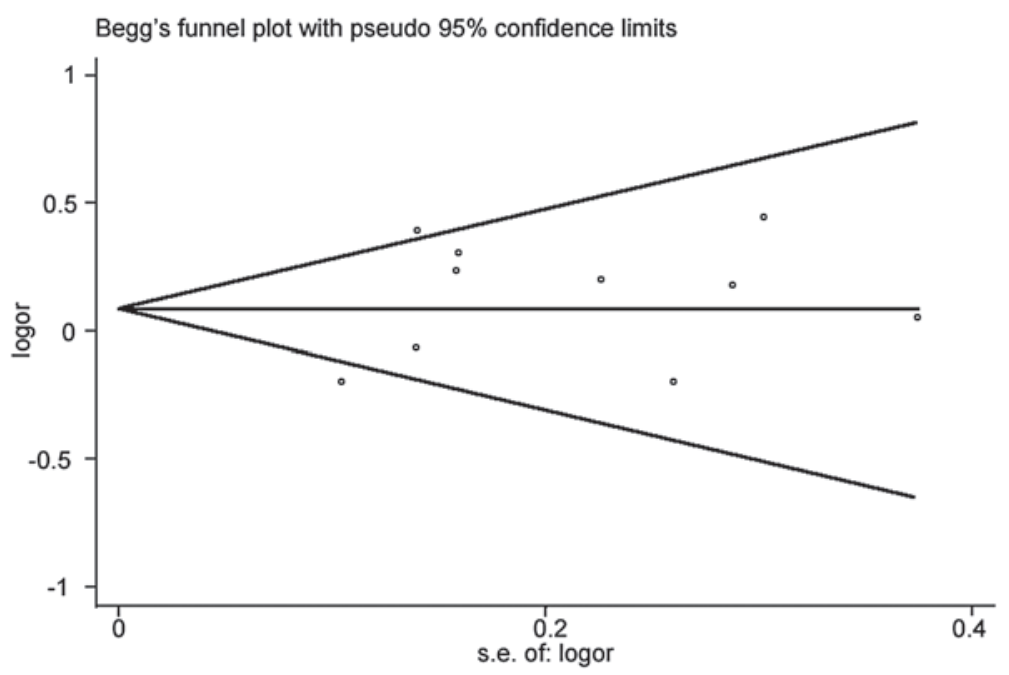

Figure 3. Funnel plot of the association of FAS-670 genotype polymorphism with cervical carcinogenesis. In Begg's test, $\mathrm{z}=0.18$, (continuity corrected), $\mathrm{P}>|\mathrm{z}|=0.858$ (continuity corrected). In Egger's test, $\mathrm{t}=1.01, \mathrm{P}>|\mathrm{t}|=0.343,(95 \% \mathrm{CI}:-1.651001-4.215824)$.

In the meta-analysis, heterogeneity was always estimated in a statistical analysis. However, the tests appeared to be of low statistical power. Thus, a subgroup meta-analysis was conducted based on ethnicity and sample size. In the ethnicity subgroups, a positive association between FAS-670 polymorphism and cervical cancer was observed in Asian, but not in Caucasian populations. However, the negative result in Caucasian must be assessed with caution, as the relatively high between-study heterogeneity may due to a mixture of populations of different races and from different geographical regions. Moreover, regarding sample size subgroups, no association between FAS-670 polymorphism and cervical cancer was observed in the smaller or in the larger size subgroups.

Regarding our results, several limitations must be mentioned. First, of all the eligible studies, there was inherent bias in the study design. Selection bias is a possible major source of heterogeneity in the acquisition of cancer samples and hospital controls. Moreover, only two studies $(27,30)$ matched the number of subjects between the case and the control groups and this lack of symmetry in the included 
subjects may lead to deviations. All these factors may result in bias.

Second, the pathological classification and clinical stages were not consistent. For example, some of the studies only included samples of cervical cancer, whereas others included cervical cancer and LSIL or HSIL. The potential deviation may produce different outcomes.

Finally, the combined analysis of different ages and races may lead to deviations. The mean age range of the eligible subjects was 29-55 years in the case and control groups. However, one study reported that the risk of cervical cancer increases with advancing age (23). Thus, age may be the cause of heterogeneity. Moreover, the incidence of cervical cancer differs among different ethnicities $(14,17,18)$; therefore, a subgroup analysis according to race is required. However, our meta-analysis was only focused on Asians and Caucasians, which may have affected the outcome of the ethnicity subgroup analysis.

\section{References}

1. Zörnig M, Hueber A, Baum W and Evan G: Apoptosis regulators and their role in tumorigenesis. Biochim Biophys Acta 1551: F1-F37, 2001.

2. Thompson CB: Apoptosis in the pathogenesis and treatment of disease. Science 267: 1456-1462, 1995.

3. Evan GI and Vousden KH: Proliferation, cell cycle and apoptosis in cancer. Nature 411: 342-348, 2001.

4. Lorenz HM, Herrmann M, Winkler T, Gaipl U and Kalden JR: Role of apoptosis in autoimmunity. Apoptosis 5: 443-449, 2000.

5. Itoh N, Yonehara S, Ishii A, et al: The polypeptide encoded by the cDNA for human cell surface antigen Fas can mediate apoptosis. Cell 66: 233-243, 1991.

6. Oehm A, Behrmann I, Falk W, et al: Purification and molecular cloning of the APO-1 cell surface antigen, a member of the tumor necrosis factor/nerve growth factor receptor superfamily. Sequence identity with the Fas antigen. J Biol Chem 267 10709-10715, 1992.

7. Nagata S: Apoptosis by death factor. Cell 88: 355-365, 1997.

8. Butler LM,HewettPJ, Butler WJ and Cowled PA: Down-regulation of Fas gene expression in colon cancer is not a result of allelic loss or gene rearrangement. Br J Cancer 77: 1454-1459, 1998.

9. Lee SH, Shin MS, Park WS, et al: Alterations of Fas (APO-1/CD95) gene in transitional cell carcinomas of urinary bladder. Cancer Res 59: 3068-3072, 1999.

10. Shimonishi T, Isse K, Shibata F, et al: Up-regulation of Fas ligand at early stages and down-regulation of Fas at progressed stages of intrahepatic cholangiocarcinoma reflect evasion from immune surveillance. Hepatology 32: 761-769, 2000.

11. Nunobiki O, Ueda M, Toji E, et al: Genetic polymorphism of cancer susceptibility genes and HPV infection in cervical carcinogenesis. Patholog Res Int 2011: 364069, 2011.

12. Huang QR, Morris D and Manolios N: Identification and characterization of polymorphisms in the promoter region of the human Apo-1/Fas (CD95) gene. Mol Immunol 34: 577-582, 1997.

13. Sun T, Zhou Y, Li H, et al: FASL -844C polymorphism is associated with increased activation-induced T cell death and risk of cervical cancer. J Exp Med 202: 967-974, 2005.

14. Kordi Tamandani DM, Sobti RC and Shekari M: Association of Fas-670 gene polymorphism with risk of cervical cancer in North Indian population. Clin Exp Obstet Gynecol 35: 183-186, 2008.

15. Scarinci IC, Garcia FA, Kobetz E, et al: Cervical cancer prevention: new tools and old barriers. Cancer 116: 2531-2542, 2010.

16. Anorlu RI: Cervical cancer: the sub-Saharan African perspective. Reprod Health Matters 16: 41-49, 2008.

17. Moore MA, Manan AA, Chow KY, et al: Cancer epidemiology and control in peninsular and island South-East Asia - past, present and future. Asian Pac J Cancer Prev 2: 81-98, 2010.
18. Langmár Z, Németh M and Kornya L: Cervical cancer screening in Hungary - epidemiologic, historical and methodologic aspects. Orv Hetil 152: 2063-2066, 2010 (In Hungarian).

19. Parkin DM, Sitas F, Chirenje M, Stein L, Abratt R and Wabinga H: Part I: Cancer in indigenous Africans - burden, distribution, and trends. Lancet Oncol 9: 683-692, 2008.

20. Guan P, Howell-Jones R, Li N, Bruni L, de Sanjosé S, Franceschi S and Clifford GM: Human papillomavirus types in 115,789 HPV-positive women: a meta-analysis from cervical infection to cancer. Int J Cancer 131: 2349-2359, 2012.

21. Pate Capps N, Stewart A and Burns C: The interplay between secondhand cigarette smoke, genetics, and cervical cancer: a review of the literature. Biol Res Nurs 10: 392-399, 2009.

22. Dikshit R, Gupta PC, Ramasundarahettige C, et al: Cancer mortality in India: a nationally representative survey. Lancet 12 : 1807-1816, 2012.

23. Zucchi F, da Silva ID, Ribalta JC, et al: Fas/CD95 promoter polymorphism gene and its relationship with cervical carcinoma. Eur J Gynaecol Oncol 30: 142-144, 2009.

24. Kang S, Dong SM, Seo SS, Kim JW and Park SY: FAS -1377 G/A polymorphism and the risk of lymph node metastasis in cervical cancer. Cancer Genet Cytogenet J 180: 1-5, 2008.

25. Ueda M, Terai Y, Kanda K, et al: Fas gene promoter -670 polymorphism in gynecological cancer. Int J Gynecol Cancer 16 (Suppl 1): 179-182, 2006.

26. Zoodsma M, Nolte IM, Schipper M, et al: Interleukin-10 and Fas polymorphisms and susceptibility for (pre)neoplastic cervical disease. Int J Gynecol Cancer 3: 282-290, 2005.

27. Lai HC, Lin WY, Lin YW, Chang CC, Yu MH, Chen CC and Chu TY: Genetic polymorphisms of FAS and FASL (CD95/CD95L) genes in cervical carcinogenesis: an analysis of haplotype and gene-gene interaction. Gynecol Oncol 99: 113-118, 2005.

28. Ueda M, Hung YC, Terai Y, et al: Fas gene promoter -670 polymorphism (A/G) is associated with cervical carcinogenesis. Gynecol Oncol 98: 129-133, 2005.

29. Dybikowska A, Sliwinski W, Emerich J and Podhajska AJ: Evaluation of Fas gene promoter polymorphism in cervical cancer patients. Int J Mol Med 14: 475-478, 2004.

30. Lai HC, Sytwu HK, Sun CA, et al: Single nucleotide polymorphism at Fas promoter is associated with cervical carcinogenesis. Int J Cancer 103: 221-225, 2003.

31. Chatterjee K, Engelmark M, Gyllensten U, et al: Fas and FasL gene polymorphisms are not associated with cervical cancer but differ among black and mixed-ancestry South Africans. BMC Res Notes 26: 238, 2009.

32. Zhang Z, Xue H, Gong W, Wang M, Yuan L, Han S and Zhang Z: FAS promoter polymorphisms and cancer risk: a meta-analysis based on 34 case-control studies. Carcinogenesis 30: 487-493, 2009.

33. Weir BS (ed): Genetic data analysis II: Methods for discrete population genetic data. Sunderland Mass: Sinauer Associates, 1996.

34. Higgins JP, Thompson SG, Deeks JJ and Altman DG: Measuring inconsistency in meta-analyses. BMJ 327: 557-560, 2003.

35. Zintzaras E, Chatzoulis DZ, Karabatsas CH and Stefanidis I: The relationship between C677T methylenetetrahydrofolate reductase gene polymorphism and retinopathy in type 2 diabetes: a meta-analysis. J Hum Genet 50: 267-275, 2005.

36. Begg CB and Mazumdar M: Operating characteristics of a rank correlation test for publication bias. Biometrics 50: 1088-1101, 1994.

37. Engelmark MT, Renkema KY and Gyllensten UB: No evidence of the involvement of the Fas -670 promoter polymorphism in cervical cancer in situ. Int J Cancer 112: 1084-1085, 2004.

38. Lerma E, Romero M, Gallardo A, et al: Prognostic significance of the Fas-receptor/Fas-ligand system in cervical squamous cell carcinoma. Virchows Arch 452: 65-74, 2008.

39. Li H, Guo HY, Sun T, Zhou YF, Lin DX, Zhang WH and Qiao J: Association between Fas/Fas L genes promoter polymorphisms and pathogenic risk of cervical cancer. Chin J Oncol 31: 38-41, 2009 (In Chinese). 